\title{
Asistencia ventricular derecha con bomba centrífuga
}

\author{
O neglio Pedemonte $V^{1}$, Ernesto Aránguiz Santander ${ }^{1}$, \\ H umberto Torres $\mathrm{H}^{1}$, Lorenzo Merello $\mathrm{N}^{1}$, \\ Andrés Vera $\mathrm{P}^{1}$, Rienzi Díaz $\mathbf{N}^{2}$, Jorge Kaplán $\mathbf{M}^{1}$. \\ Right ventricular assistance \\ with a centrifugal pump. \\ Report of two cases
}

Acute right ventricular failure after cardiac surgery occurring in the first postoperative hours is associated with a bad prognosis. We have used a centrifugal pump either for left, right or biventricular assistance. However, the use of this device for pure right ventricular assistance is rare. We report a 30 year-old female undergoing a mitral valve replacement and a 42 year-old male undergoing a cardiac transplantation, who had a successful right ventricular assistance using a centrifugal pump, due to a failing right ventricle, as the result of insufficient myocardial protection and severe pulmonary hypertension. These two cases illustrate the value of the mechanical ventricular assist device for the treatment of right heart failure (Rev Méd Chile 2008; 136: 359-66).

(Key w ords: Heart transplantation; Hypertension, pulmonary; Ventricular dysfunction, right)

Recibido el 5 de junio, 2007. Aceptado el 23 de julio, 2007.

${ }^{1}$ Servicio de Cirugía Cardiovascular y Unidad de Tratamiento Intensivo Cardiovascular, Hospital Dr. Gustavo Fricke. ${ }^{2}$ Unidad de Cuidados Intensivos, Clínica Reñaca, Viña del Mar, Chile.

\begin{abstract}
A pesar de los grandes avances en el manejo perioperatorio inmediato de la cirugía cardiaca, la falla cardiaca derecha refractaria a drogas es aún causa importante de morbilidad y mortali$\mathrm{dad}^{1}$

Ésta puede manifestarse en el paciente sometido a trasplante cardiaco, en los portadores de valvulopatía mitral con resistencia vascular pulmo-
\end{abstract}

Correspondencia a: Dr. Oneglio Pedemonte. Unidad de Tratamiento Intensivo Cardiovascular, Hospital Dr. Gustavo Fricke. Calle Alvarez 1532, Viña del Mar, Chile. Fono: 322652338. Fax: 32-2652434.

E mail: onegliopedemonte@hotmail.com nar (RVP) elevada operados tardíamente, o en pacientes con cardiopatía terminal que han requerido de asistencia ventricular izquierda ${ }^{1,2}$.

La insuficiencia cardiaca derecha aguda es una entidad compleja y dramática. Sus consecuencias son el bajo débito con repercusión hemodinámica catastrófica, que de no mediar intervenciones excepcionales provocan un colapso hemodinámico que llevará al paciente irremediablemente a la muerte 3,4 .

Cuando se han agotado las posibilidades terapéuticas con drogas vasoactivas, inhibidores de fosfodiesterasa III, vasodilatadores pulmonares específicos como prostaglandinas y óxido nítrico, es vital recurrir al uso de asistencia ventricular mecánica ${ }^{1-5}$. 
En esta ocasión comunicamos los casos clínicos de dos pacientes en los que, por distintas circunstancias, la falla ventricular derecha habría tenido un curso irreversible de no haberse utilizado la asistencia ventricular mecánica derecha (AVD). En el primer caso existía un aumento severo de la RVP asociada a disfunción ventricular derecha, en una paciente sometida a recambio valvular mitral y en el segundo, la falla ventricular derecha aguda ocurrió en un paciente receptor de un trasplante cardiaco ortotópico, como consecuencia de una RVP severamente aumentada en combinación a un injerto implantado deficiente e incapaz de soportar el estrés hemodinámico.

Este tipo de situaciones clínicas, particularmente complejas y difíciles de manejar, han sido escasamente publicadas en la literatura, razón por la que consideramos de interés comunicarlas.

\section{Caso Clínico 1}

Mujer de 30 años, portadora de estenosis mitral e hipertensión pulmonar severa, quien fue sometida a reemplazo valvular mitral. El área valvular mitral era de $0,7 \mathrm{~cm}^{2}$ y la presión sistólica de arteria pulmonar $65 \mathrm{mmHg}$. La intervención quirúrgica se realizó bajo hipotermia moderada $\left(30^{\circ} \mathrm{C}\right)$, con un pinzamiento aórtico de $48 \mathrm{~min}$, implantándose una prótesis mecánica Starr-Macchi N³0. Durante la circulación extracorpórea (CEC) llamó la atención la dificultad para detener la actividad eléctrica del corazón, a pesar del uso de cardioplegia cristaloidea cada $20 \mathrm{~min}$. Al término de la cirugía se objetivó un bajo débito cardiaco con un índice cardiaco (IC) de $2,1 / 1 \mathrm{t} / \mathrm{m}^{2}$, una presión arterial media (PAM) de $62 \mathrm{mmHg}$ y una presión de capilar pulmonar (CAP) de $20 \mathrm{mmHg}$. Por esto se inició tratamiento con drogas vasoactivas: adrenalina $0,1 \mathrm{mcg} / \mathrm{kg} / \mathrm{min}$, noradrenalina $0,28 \mathrm{mcg} / \mathrm{kg} /$ min, y amrinona $2 \mathrm{mcg} / \mathrm{kg} / \mathrm{min}$. A pesar del tratamiento iniciado, la condición hemodinámica de la paciente empeoró, desecandenándose un síndrome de respuesta inflamatoria que rápidamente provocó una falla orgánica de otros sistemas, destacando la fiebre alta, leucocitosis y lactacidemia de $100 \mathrm{U} / \mathrm{lt}$, sin foco séptico demostrado, iniciándose un tratamiento antibiótico empírico de amplio espectro asociando imipenem y sulbactam-ampicilina. Paralelamente, aparecieron otras fallas de órganos: 1) insuficiencia respiratoria aguda con alto requerimiento de oxígeno y PEEP; 2) coagulopatía de consumo, con recuento de plaquetas de $22.000 / \mathrm{mm}^{3}$, que requirió transfusiones masivas de factores de coagulación y concentrados plaquetarios; 3) insuficiencia renal aguda; 4) isquemia miocárdica con elevación de enzimas cardiacas (CK-MB 77 U/lt).

A las $36 \mathrm{~h}$ del postoperatorio, el débito cardíaco había empeorado y la situación clínica era muy crítica, razón por lo que decidimos el uso de la AVD.

Inicialmente, se utilizaron débitos de hasta 5 lt/min que no fueron soportados por el ventrículo izquierdo, desencadenándose un edema agudo del pulmón que se corrigió bajándolo a 3,7lt/min, obtenidos con 1.500 revoluciones por min (RPM). Este gasto se mantuvo durante $48 \mathrm{~h}$, lo que permitió el control de la falla multiorgánica y la disminución de las drogas inotrópicas (Figura 1). Al tercer día de AVD, se inició el retiro progresivo del sistema de asistencia ventricular y, luego de lograrse una hemodinamia satisfactoria, se retiró completamente el circuito implantado después de $111 \mathrm{~h}$ de asistencia ventricular derecha (Figura 2). No se observaron incidentes relacionados con el procedimiento, sin embargo, la paciente presentó algunas complicaciones producto de su falla multiorgánica, entre las que destacó una coagulopatía severa que al duodécimo día postoperatorio, provocó una hemorragia digestiva alta secundaria a una duodenitis erosiva y un hemoperitoneo espontáneo, que se manejó con lavado peritoneal percutáneo más aportes de factores de coagulación. Al día 14 del postoperatorio, la paciente presentó un taponamiento cardiaco que requirió de drenaje percutáneo de $600 \mathrm{ml}$ de sangre, lo que resolvió satisfactoriamente la complicación. Al vigésimo día del postoperatorio, la paciente fue retirada del ventilador mecánico.

La falla renal por necrosis tubular aguda, mantuvo a la paciente en hemodiálisis durante 6 semanas. Fue dada de alta a los 65 días del postoperatorio, en buenas condiciones generales y en capacidad funcional (CF) II. La paciente fue derivada a su hospital de origen donde continuó su control y tratamiento. Actualmente, a los 15 años del reemplazo mitral y del procedimiento descrito, la paciente se encuentra en CF I y en 


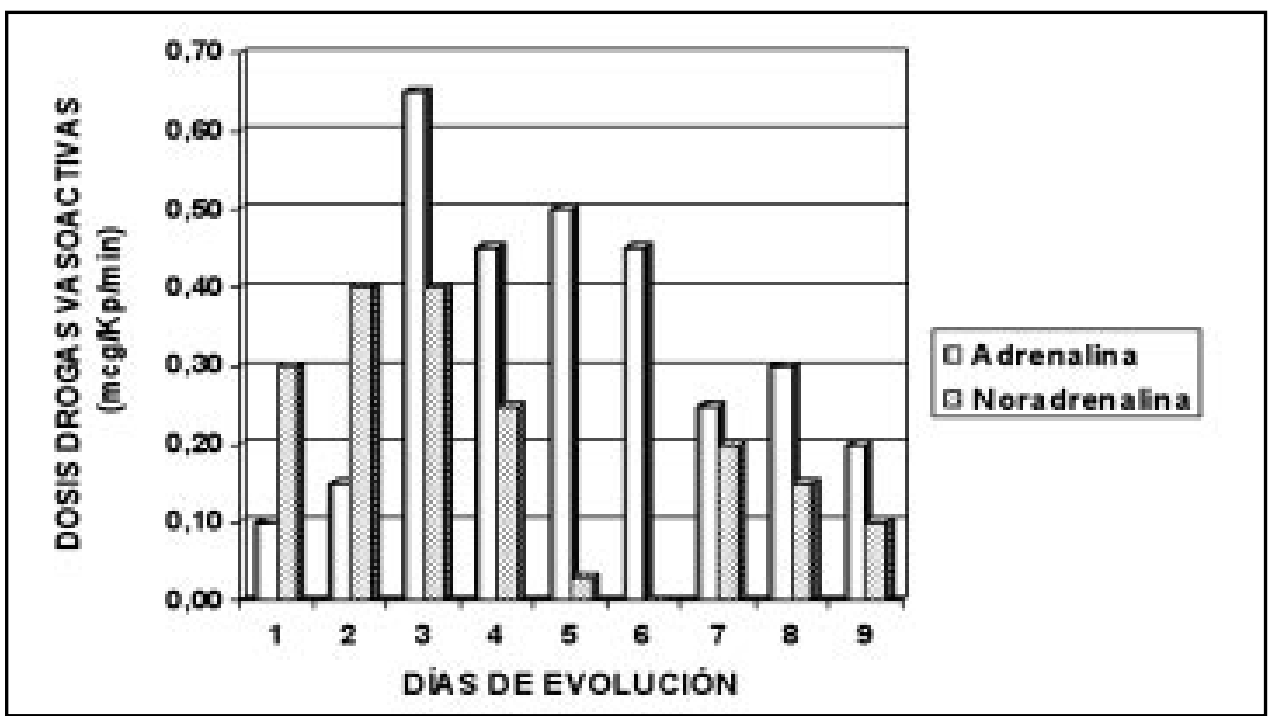

Figura 1. Dosis de adrenalina y noradrenalina en relación a los días de evolución (Caso 1).

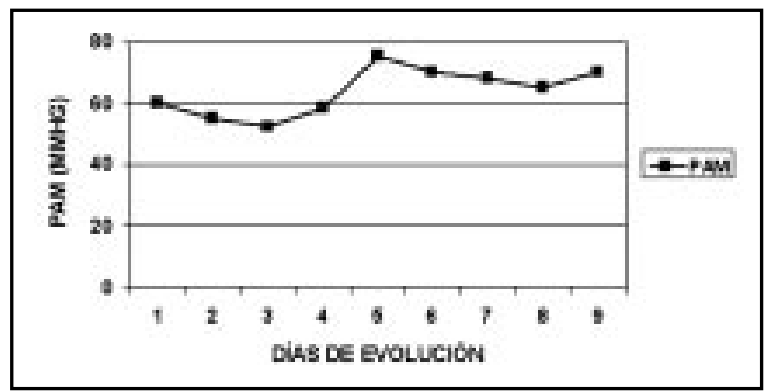

Figura 2. Presión arterial media (PAM) en relación a los días de evolución (Caso 1).

terapia anticoagulante como único tratamiento. Un ecocardiograma bidimensional y Doppler color realizado en abril de 2007, demostró un ventrículo izquierdo con fracción de eyección (FE) de $62 \%$, y un ventrículo derecho de tamaño y contractilidad normal. La presión sistólica de arteria pulmonar fue estimada en $30 \mathrm{mmHg}$.

\section{Caso CLÍNICO 2}

Varón de 42 años con historia de insuficiencia cardiaca terminal secundaria a una miocardiopatía dilatada idiopática diagnosticada el año 1998, con un ventrículo izquierdo severamente dilatado, una $\mathrm{FE}$ de $20 \%$ y en tratamiento con enalapril, espironolactona, carvedilol, hidroclorotiazida, digoxina y aceno- cumarol, terapia con la que se mantuvo en CF I hasta fines de 2005. El sondeo cardíaco derecho demostró una resistencia vascular pulmonar de 2,1 unidades Wood (UW). En enero de 2006, sufrió una muerte súbita por una taquicardia ventricular que degeneró en una fibrilación ventricular, de la cual fue reanimado exitosamente. En esas condiciones fue trasladado a nuestro centro bajo infusión de amiodarona endovenosa. Debido a su cardiopatía y su muy mala función ventricular, se incorporó a la lista de espera para trasplante cardiaco. Durante su periodo de espera de un donante, se le implantó un cardiodesfibrilador bicameral. En los siguientes meses, el paciente presentó un importante deterioro clínico que lo llevó a una CF III. En abril de 2006, se realizó un nuevo cateterismo derecho que reveló un aumento de la RVP a 3,7 UW, alcanzando 7,3 UW en 
agosto de 2006, que determinó adicionar sildenafil al tratamiento ( $25 \mathrm{mg}$ cada 8 h). Quince días más tarde, se repitió el estudio hemodinámico para evaluar reversibilidad. La RVP basal era 4,3 UW, que disminuyó a 2,26 UW con la infusión de nitroprusiato sódico. El paciente fue trasplantado en octubre de 2006. El tiempo de CEC fue de $215 \mathrm{~min}$, con un tiempo total de isquemia del injerto de $178 \mathrm{~min}$. Al retiro de la CEC, se evidenció una hipertensión pulmonar severa que provocó una falla ventricular derecha catastrófica, que requirió de tratamiento con inótropos, vasopresores, balón de contrapulsación aórtico (BCPA) y, finalmente, AVD con bomba centrífuga.

Durante el período postoperatorio inmediato en la Unidad de Tratamiento Intensivo Cardiovascular (UTICV), requirió de dosis máximas de vasopresores: noradrenalina $0,1 \mathrm{mcg} / \mathrm{kl} / \mathrm{min}$; adrenalina 0,16 $\mathrm{mcg} / \mathrm{kl} / \mathrm{min}$; dopamina $5,7 \mathrm{mcg} / \mathrm{kg} / \mathrm{min}$ ). El BCPA se mantuvo 1:1 y la AVD entre 2.000 a 2.500 RPM. Se administró, además, levosimendan $0,1 \mathrm{mcg} / \mathrm{kg} /$ min durante $24 \mathrm{~h}$ en infusión continua; prostaglandina $0,01 \mathrm{mcg} / \mathrm{kg} / \mathrm{min}$; sildenafil $50 \mathrm{mg}$ por vía orogástrica. El tratamiento descrito se mantuvo inalterable durante las primeras $72 \mathrm{~h}$, lo que permitió una mejoría de la condición hemodinámica (Figura 3), por lo que se pudo lograr el retiro del apoyo inotrópico (Figura 4).

A las $48 \mathrm{~h}$ del postoperatorio, se inició el retiro progresivo de la AVD, disminuyendo el apoyo de 2.000 a 1.400 RPM. Al tercer día, se logró reducir de 1.400 a 1.000 RPM (Figura 5). La AVD, fue retirada al $5^{\circ}$ día, quirúrgicamente, en pabellón. La progresiva estabilización clínica del paciente permitió el retiro del BCPA al $6^{\circ}$ día y su extubación de AVD al día $7^{\circ}$. Al $9^{\circ}$ día postrasplante cardiaco,

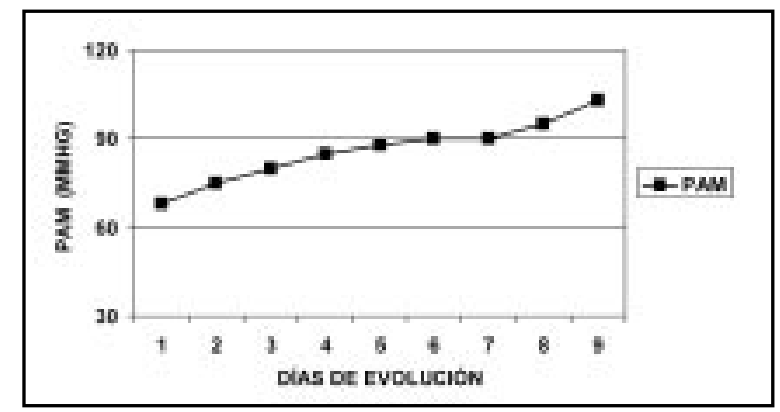

Figura 3. Presión arterial media (PAM) según días de evolución (Caso 2).

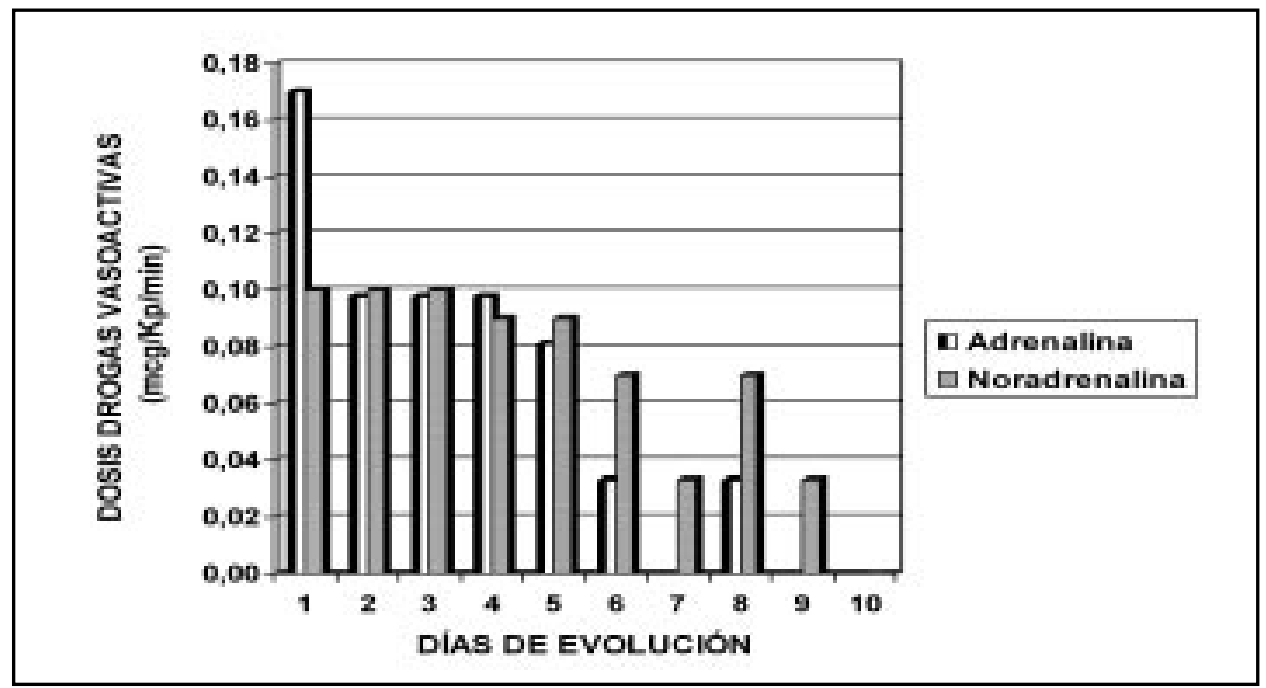

Figura 4. Dosis de adrenalina y noradrenalina en relación a los días de evolución (Caso 2). 


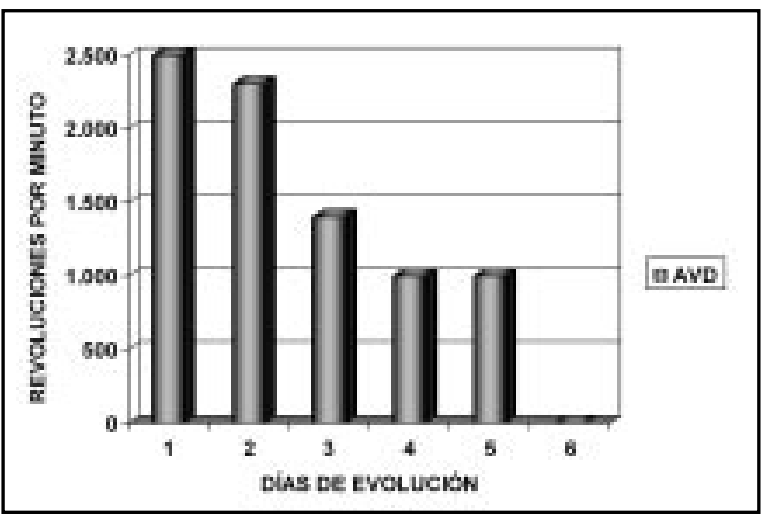

Figura 5. Revoluciones por minuto del cono de la asistencia ventricular derecha (AVD) del primer día del procedimiento hasta el destete de la asistencia.

el paciente egresó de la UTICV a la sala de Cuidados Intermedios Cardiovascular, con tratamiento oral. A la $3^{\mathrm{a}}$ semana, el paciente fue dado de alta hospitalaria en CF I, sin hipertensión pulmonar ni falla ventricular derecha.

DESCRIPCIÓN DE LA TÉCNICA DE ASISTENCIA VENTRICULAR MECÁNICA DERECHA

En ambos casos, la técnica de AVD se realizó conectando quirúrgicamente una cánula Pacífico № 24 en la aurícula derecha (AD) y una cánula Argyle curva № 22 en el tronco de la arteria pulmonar. Estas fueron exteriorizadas por la esternotomía (Figura 6).

El circuito extracorpóreo fue conectado al cono de la centrífuga a través de 2 extensiones de $120 \mathrm{~cm}$ de largo, permitiendo de esta manera aspirar la sangre de la $\mathrm{AD}$ para impulsarla hacia la arteria pulmonar, gracias al efecto vortex provocado por las aspas del cono (Figura 7). El flujo óptimo entregado por la centrífuga se pudo obtener con la medición del débito cardiaco por Doppler, así como por el ajuste de las RPM del sistema. Posterior al procedimiento, ambos pacientes fueron trasladados a la UTICV donde

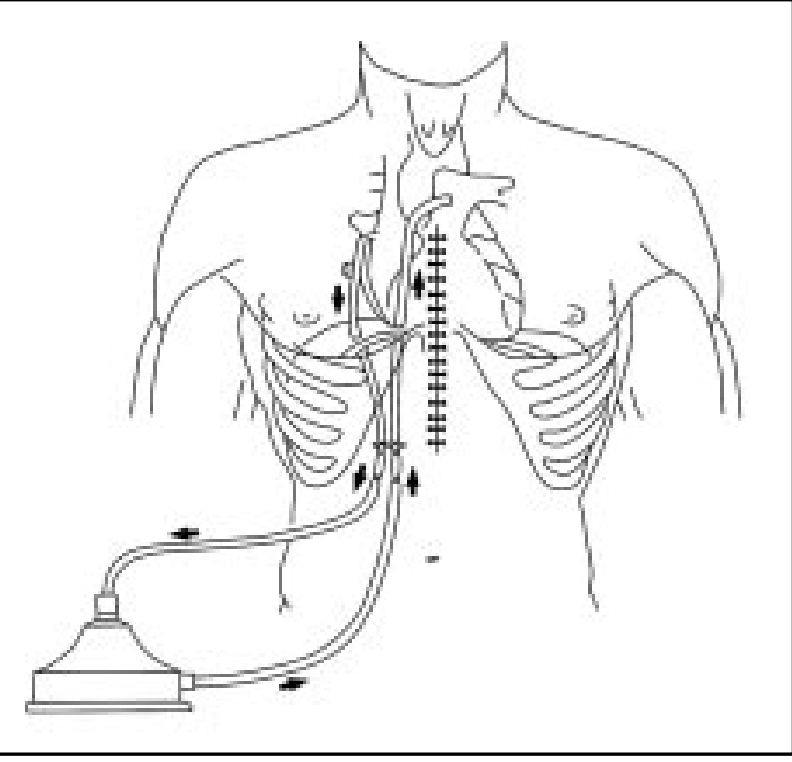

Figura 6. Técnica de asistencia ventricular derecha (AVD), en la cual se aprecia aspiración de la sangre aurícula derecha y la impulsión a través del cono a la arteria pulmonar. 


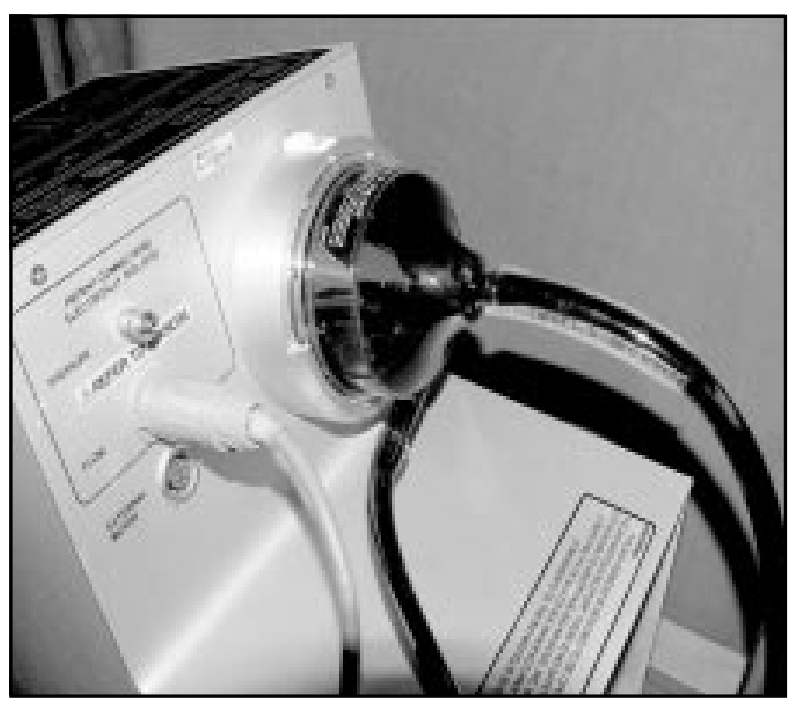

Figura 7. Bomba centrífuga en funcionamiento con su sistema de cánulas de conexión y cono de aspas de acrílico que giran para aspirar e impulsar la sangre.

fueron vigilados estrechamente para el adecuado control del débito entregado por la centrífuga y ajustar las drogas inotrópicas, de acuerdo a la respuesta hemodinámica obtenida. El tiempo parcial de tromboplastina (TTPK) se mantuvo entre 50 y $70 \mathrm{~s}$, con el objetivo de evitar hemorragias postoperatorias. Las bombas centrífugas empleadas fueron la Sarns, modelo Delphin (3 M, Ann Arbor, Mi) en el primer caso, y la Biomedicus (Eden Prairie MN) en el segundo.

\section{DISCUSIÓN}

La falla ventricular derecha aguda presentada en los dos casos clínicos descritos, corresponde al llamado síndrome postcardiotomía, el que se caracteriza por disfunción cardiaca uni o biventricular posterior al retiro de la $\mathrm{CEC}^{1}$.

Apesar de los importantes progresos en materia de protección miocárdica, la necesidad de apoyo mecánico post CEC, tiene una incidencia de 1\%-6\% en pacientes sometidos a cirugía cardiaca ${ }^{1}$.

La falla cardiaca aguda se puede producir por depleción de los fosfatos energéticos del miocardio insuficiente o dilatado, o bien, por un aumento del consumo de sustratos productores de energía. Esto último puede ocurrir en un ventrículo derecho hipertrófico y dilatado, como ocurrió en nuestro primer paciente, o por un ventrículo derecho atontado como consecuencia de un tiempo de isquemia prolongado, situación representada por el segundo. En ambos casos, el mecanismo patológico común es una protección miocárdica insuficiente asociada a una postcarga derecha aumentada, producto de un marcado aumento de la $\mathrm{RVP}^{2}$.

La AVD usada en ambos casos correspondió a dos máquinas centrífugas técnicamente similares, con un sistema de aspas de acrílico que rotan a gran velocidad (entre 1.000 y 10.000 RPM), creando un efecto vortex que impulsa la sangre hasta los límites deseados 3 . Este es un sistema que está disponible desde hace muchos años y tiene la ventaja de no producir un daño significativo sobre los glóbulos rojos y otros elementos sanguíneos, pero que requiere de anticoagulación que para este tipo de pacientes puede ser muy deletéreo (aumento del riesgo de sangrado y necesidad de reexploraciones). Por lo tanto, durante su utilización es fundamental ajustar la dosis de heparina de manera muy precisa, evitando que el TTPK supere los $70 \mathrm{~s}$ para evitar un sangrado importante. Por otra parte, esta técnica no debe utilizarse por más de 10 días, ya que condiciona una 
respuesta inflamatoria que progresa con el tiempo de uso, provocando fallas en otros órganos. Por esta razón, su utilización está indicada en pacientes que requieren una asistencia ventricular mecánica de corta duración en los que exista, además, una causa potencialmente reversible ${ }^{1}$.

La falla cardiaca aguda relacionada con la cirugía sobre la válvula mitral es cada vez más inusual debido a la precocidad en el diagnóstico de la patología mitral que condiciona una intervención quirúrgica más oportuna, pero también debido a los avances en la protección miocárdica y al avance sustantivo en el manejo perioperatorio de estos pacientes ${ }^{6-9}$. Sin embargo, en nuestro país aún existen portadores de valvulopatía mitral de larga evolución con RVP elevadas que se operan tardíamente. Cuando la hipertensión pulmonar es severa, constituye un factor que se asocia a aumento en la morbimortalidad. La remisión de la RVP posterior a la cirugía valvular mitral no es inmediata, lo que puede condicionar bajo débito, insuficiencia cardiaca derecha crónica y también la muerte ${ }^{10-12}$. En nuestra primera paciente, la AVD permitió superar con éxito la falla cardiaca derecha, recuperar la función del ventrículo derecho y normalizar la RVP, lo que explica porqué la paciente se encuentra realizando una vida normal, quince años después de la cirugía valvular mitral.

Nuestro paciente trasplantado era portador de una disfunción ventricular severa de varios años de evolución que presentó una descompensación aguda, producto de una arritmia ventricular compleja. La evaluación inicial objetivó hipertensión pulmonar con cifras que contraindicaban el trasplante cardiaco. Sin embargo, sometido a una prueba de reversibilidad con vasodilatadores, se logró una significativa disminución de la RVP, alcanzándose cifras <4 UW, que permitieron su incorporación a la lista de espera para trasplante

\section{REFERENCIAS}

1. Naka Y, Chen J, Rose E. Assisted Circulation in the Treatment of Heart Failure. En: Zipes D, Libby P, Bonow R, Braunwald E. Braunwald's Heart Disease, $7^{\text {th }}$ Edition, 2005.

2. Kavarana M, Pessi-Minsley S, Urtecho J, Catanese K, FlanNery M, Oz M, NaKa Y. Right ventricular cardiaco, el que se realizó luego de 9 meses de espera. Los pacientes con insuficiencia cardiaca terminal y aumento severo de la RVP tienen morbimortalidad elevada ${ }^{13,14}$, sin embargo, aquellos con hipertensión pulmonar reversible, que bajo terapia con vasodilatadores alcanzan una RVP $<2,5$ UW y gradiente transpulmonar $<12$ $\mathrm{mmHg}$, si bien tienen una mayor mortalidad perioperatoria, no presentan diferencias significativas en la sobrevida a largo plazo ${ }^{13-15}$. De cualquier modo, estos pacientes presentan un riesgo mayor de desarrollar insuficiencia cardiaca derecha prolongada, la que puede presentarse incluso luego de los primeros 30 días del postoperatorio. Por lo tanto, es recomendable ser más agresivo en el manejo perioperatorio de la RVP, usando vasodilatadores pulmonares específicos, como prostaglandinas, óxido nítrico, isorsorbide o sildenafil $6,16,17$.

Otro de los factores que agravó la falla cardiaca derecha en nuestro paciente trasplantado fue el desequilibrio entre la RVP del receptor versus la mala calidad del injerto, a juicio del cirujano procurador; esta situación, que determinó dificultades para el retiro de la CEC, sólo pudo lograrse con apoyo inotrópico múltiple, que incluyó el uso de levosimendam, fármaco nuevo con propiedades inótropas y vasodilatadoras que no acarrea aumento del consumo de $\mathrm{O}_{2}$ y que ha demostrado su eficacia en el tratamiento de pacientes con falla cardiaca aguda ${ }^{18}$.

\section{Conclusión}

Los dos casos presentados en la presente comunicación, muestran el valor de la AVD realizada con bomba centrífuga, en pacientes post cirugía cardiaca que cursan con falla cardiaca derecha severa, sin respuesta a la terapia convencional.

dysfunction and organ failure in left ventricular assist devices recipients: A continuing problem. Ann Thorac Surg 2002; 73: 745-50.

3. Pedemonte O, Aranguiz E, Nahmías M, Torres $H$, Kaplan J. Asistencia ventricular derecha con bomba centrífuga. Bol Hosp Viña del Mar, 1993; 49 35-9.

4. Pedemonte O, Torres H, De Prada Mt, Cortez G, Barrios JP, MuÑoz JA et al. Asistencia circulatoria con 
bomba centrífuga: Experiencia en el Hospital Dr. Gustavo Fricke. Rev Chilena Cardiol 1994; 13: 162.

5. Castro P, Baraona F, Baeza C, Mc-Nab P, Berlin A, Zalaquett R et al. Asistencia ventricular mecánica como puente al trasplante en pacientes en shock cardiogénico: Experiencia preliminar en Chile con ABIOMED BVS $5000^{\circledR}$. Rev Méd Chile 2006; 134: 1019-23.

6. Beck JR, Mongero LB, Kroslowitz RM, Choudhri AF, Chen JM, Derose JJ. Inhaled nitric oxide improves hemodynamics in patients with acute pulmonary hypertension after high risk cardiac surgery. Perfusion 1999; 14: 37-42.

7. D'ambra mn, la Raia PJ, Philbin DM, Hilgenberg AD, Buckley MJ. Prostaglandin E1. A new therapy for refractory right heart failure and pulmonary hypertension after MVR. J Thorac Cardiovasc Surg 1985; 89: 567.

8. Goenen M, Pedemonte O, Baele P, Col J. Amrinona in the management of low cardiac output after open-heart surgery. Am J Cardiol 1985; (abstract) 56: 33B.

9. Pedemonte O, Goenen M, Baele P, Kestens-Servaye Y. Aporte de la Amrinona en el tratamiento del bajo débito después de la cirugía cardiaca. Revista Chilena de Anestesia 1985; 14: 155-60.

10. Jackson JM, Thomas SJ. Valvular Heart Disease, En: Cardiac Anesthesia. Kaplan JA, Reich DL, Konstadt SN, eds. Philadelphia: WB Saunders, 1999; 72784.

11. Vincens J, Temizer D, Post J, Edmunds He, Herrmann HC. Long-term outcome of cardiac surgery in patient with mitral stenosis and severe pulmonary hypertension. Circulation 1995; 92: 137-42.

12. Georgeson S, Ioannis P, Kleaveland JP, Heilbrunn S, GonZALEz R. Effect of percutaneous ballon valvu- loplasty on pulmonary hypertension in mitral stenosis. Am Heart J 1993; 125: 1374-9.

13. Kirklin JK, Naftel DC, Blackstone EH, WhiteWilliams C, Bourge RC. Pulmonary vascular resistance and the risk of heart transplantation. J Heart Trasplant 1988; 7: 331-6.

14. Klots S, Wenzelburger F, Stypmann J, Welp H, Dress G, Scmid C, Sheld HH. Reversible pulmonary hypertension in heart transplants candidates. To transplant or not to transplant. Ann Thorac Surg 2006; 82: 1770-3.

15. Chen JM, Levin HR, Michler RE, Prusmack CJ, Rose EA, Aaronson KD. Reevaluating the significance of pulmonary hypertension before cardiac transplantation: determination of optimal thresholds and quantification of the effect of reversibility on perioperative mortality. J Thorac Cardiovasc Surg 1997; 114: 627-34.

16. Barahona F, Castro P, Pérez O, Morán S, ZalaQuetT R, Irarrázabal M, Jalil J. Utilidad del Isosorbide sublingual en la reversibilidad de la hipertensión pulmonar reactiva, en pacientes candidatos a trasplante cardiaco. Rev Méd Chile 2006; 134: 201-6.

17. Fullerton D, Jones SD, Jaggers J, Piedalue F, Grover F, Mcintyre C. Effective control of pulmonary vascular resistance with inhaled nitric oxide after cardiac operation. J Thorac Cardiovasc Surg 1996; 111: 753-63.

18. Follath F, Cleland J GF, Just H, Sholtz H, Peurkurinen K, Harjola VP, Mitrovic V. Efficacy and safety of intravenous levosimendan compared with dobutamine in severe low-output heart failure (the LIDO study): A randomized doubleblind trial. Lancet 2002; 360: 196-202. 